\title{
A Novel Method to Determine Dynamic Temperature Trends Applied to In-Shoe Temperature Data During Walking
}

\author{
L. Cutajar, O. Falzon, S. Mizzi, A. Mizzi, I. Swaine, K. Springett*
}

\begin{abstract}
Body temperature is one of the fundamental measures considered in the assessment of health and wellbeing, with various medical conditions known to give rise to abnormal changes in temperature.

In particular, abnormal variations in dynamic temperature patterns during walking or exercise may be linked to a range of foot problems, which are of particular concern in diabetic patients.

A number of studies have investigated normative temperature patterns of a population by considering data from multiple participants and averaging results after an acclimatisation interval. In this work we demonstrate that the temperature patterns obtained using such an approach may not be truly representative of temperature changes in a population, and the averaging process adopted may yield skewed results.

In this work we propose an alternative approach to determine generic reference temperature patterns based on a minimization of root mean square differences between timeshifted versions of temperature data collected from multiple participants. The results obtained indicate that this approach can yield a general trend that is more representative of actual temperature changes across a population than conventional averaging methods. The method we propose is also shown to better capture and link the effects of underlying factors that influence dynamic temperature trends, which could in turn lead to a better understanding of underlying physiological phenomena.
\end{abstract}

\section{INTRODUCTION}

Monitoring the changes of a particular physiological factor in time is common in investigating the behavior or response of the human body to external factors. Changes in temperature are considered highly relevant for sports performance monitoring $[1,2]$, monitoring patients undergoing surgery [3], stroke patients [4], monitoring of pressure ulcer development [5, 6] and other scenarios [7]. In particular, temperature changes are considered to play an important role in tissue breakdown and ulceration [8].

A typical approach adopted in most studies is to determine normative patterns of temperature variations across a

Lucianne Cutajar is with the Department of Systems and Control Engineering, University of Malta, Msida, Malta (phone: 3562340 3665; email: lucianne.cutajar@um.edu.mt).

Owen Falzon is with the Centre for Biomedical Cybernetics, University of Malta, Msida. (e-mail: owen.falzon@um.edu.mt)

Stephen Mizzi and Anabelle Mizzi are with the Faculty of Health Sciences, University of Malta, Msida MSD 2080, Malta (e-mails: stephen.mizzi@um.edu.mt, anabelle.mizzi@um.edu.mt)

Ian Swaine is with Department of Life \& Sports Sciences, University of Greenwich, London, UK (e-mail: I.L.Swaine@ greenwich.ac.uk)

Kate Springett is with the Department of Allied Health Professions, Canterbury Christ Church University, Canterbury, UK (e-mail: kate.springett@canterbury.ac.uk) population by acquiring temperature data from multiple subjects following an acclimatisation period, and averaging the acquired data [9-11]. In [10], Zontak et al. investigate the change of temperature with time during exercise. The acquired temperature data is averaged and the mean value with time, together with maximum and minimum or standard deviation are determined. The mean and standard deviation of the acquired data across participants are then used to characterize normal temperature values. Similarly, Nardin et al. [11] considered the mean, standard deviation, minimum and maximum values in their investigation of the effect of ambient temperature and age on foot temperature. SandovalPalomares et al. [12] also consider averaging of acquired temperature signals in combination with curve fitting for monitoring microclimate in footwear.

In the studies by Shimazaki et al. [13,14], the thermal environment inside footwear was also investigated. In this case, the initial temperature values for each recording were also subtracted from the temporal signals in an attempt to better investigate trends in temperature changes.

In this work we show that the aforementioned direct averaging or baseline temperature subtraction procedures that are often adopted in the literature can lead to a significant loss of information as well as a potential distortion of the actual temperature trends in a population of interest.

We demonstrate that the absolute temperature values carry significant information and that a better outcome of temperature trends within a population can be obtained by time shifting and combining data from multiple participants and multiple sessions based on their root mean square differences. We show that the resulting temperature curves obtained using this approach can provide a better representation of actual dynamic temperature changes and can better take into account the factors influencing the observed temperature trends.

\section{METHOD}

In order to determine normative temperature trends across participants for a particular task, we propose a method based on a minimization of the root mean square difference (RMSD) between the available temperature recordings which could potentially include multiple sessions, multiple participants, and other influencing factors.

\section{A. Data Alignment Based on RMSD}

The first step of the proposed method involves computing the RMSD between each pair of temperature signals acquired across individuals, sessions and any other factors. The RMSD is also computed for each possible relative time shift between 
each pairwise combination. An array of RMSD values, $R\left(i, j, t_{0}\right)$, is thus obtained where:

$$
R\left(i, j, t_{0}\right)=\sqrt{\frac{\sum_{t=t_{1}}^{t_{2}}\left(y_{i}(t)-y_{j}\left(t-t_{0}\right)\right)^{2}}{N}}
$$

for $i=1, \ldots, K$ and $j=1, \ldots, K(i \neq j)$, where $K$ represents the total number of acquired signals (across sessions and participants). $t_{1}$ and $t_{2}$ represent start and end time of the interval over which the two signals overlap. Specifically, $t_{1}=0$ and $t_{2}=T-t_{0}$ when $t_{0}<0$, and $t_{1}=t_{0}$ and $t_{2}=T$ when $t_{0} \geq 0$. $T$ is the total number of samples in each signal and $N=t_{2}-t_{1}+1$ is the number of overlapping samples between the two signals. $R\left(i, j, t_{0}\right)$ thus represents the RMSD value computed for two signals $y_{i}(t)$ and $y_{j}(t)$ at time-shift value $t_{0}$.

The second step of the method involves a relative realignment of the acquired signals based on a minimization of the results of the RMSD array in Equation (1). This can be done by considering each signal $y_{i}(t)$ and finding the indices $j, t_{0}$ that correspond to the minimum value in $R\left(i, j, t_{0}\right)$ (excluding the case where $i=j$ ). The two signals, i.e. $y_{i}(t)$ and $y_{j}(t)$, are then aligned by shifting $y_{j}(t)$ by the corresponding value of $t_{0}$ in order to minimize their RMSD. Fig. 1 shows an example of two signals which were found to have a minimum RMSD at a time shift of 19 samples, prior and after alignment. This process can be repeated for each signal $y_{i}(t)$, finding and aligning the corresponding signal $y_{j}(t)$ to minimize the RMSD between the two signals each time.

The last step of the method involves a combination of all time-shifted signals from all the participants and sessions to extract general trends in the considered data through an
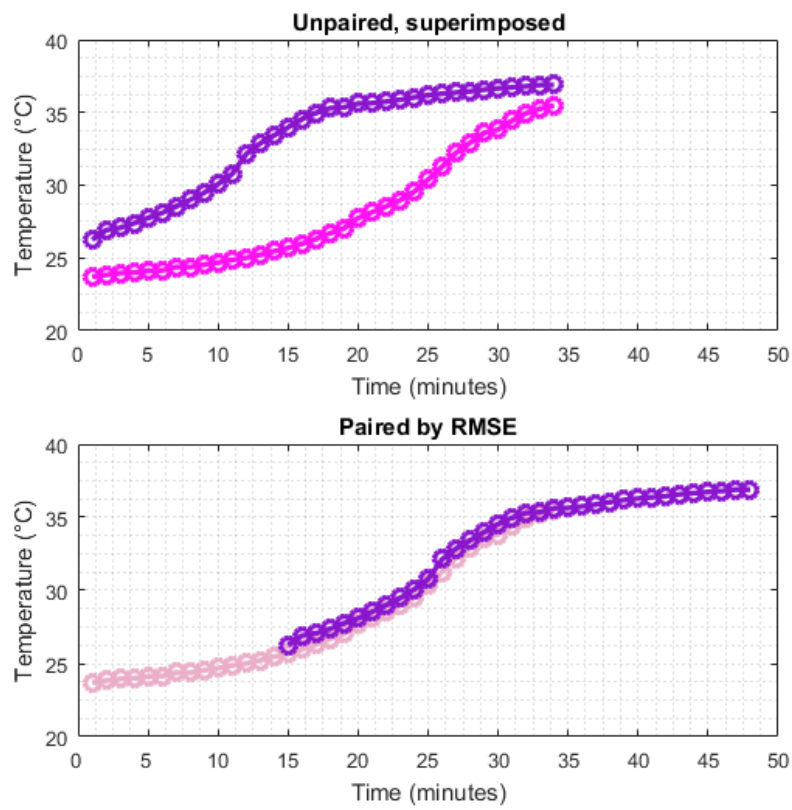

Fig. 1 (Top) Superimposed plot of temperature with time as captured in sessions 1 and 26, as plotted for conventional analysis. (Bottom) joining data from the same two sessions using RMSD to determine the least error between the two curves with respect to each other. iterative repetition of this RMSD minimization approach. The average trend obtained after applying this approach is expected to better represent general underlying physiological phenomena across participants and sessions than direct averaging of the original data.

The RMSD approach proposed above was tested on inshoe temperature measurements and the results obtained were compared to those from conventional temperature trends obtained by simple averaging procedures.

\section{B. Experimental Setup}

In order to test the proposed method, data gathered from 14 participants consisting of 5 males and 9 females, with a mean age of $49( \pm 13.3)$ years was analysed. Participants were first asked to lay in a supine position, barefoot without any plantar surface contact for 15 minutes for an acclimatisation interval. The participants were then required to walk for 40 minutes on a treadmill in a lab at what they considered a comfortable pace, generally in the range of 5 to $6 \mathrm{~km} / \mathrm{hr}$ depending on their preference. For each participant, two recording sessions were conducted, one in winter and one in summer. The study was approved by the University of Malta Research Ethics Committee.

Temperature data was gathered using an in-shoe thermistor placed close to the hallux (big toe) using a BIOPAC MP150 (BIOPAC Systems, Inc.) and AcqKnowledge Software v4.1. The temperature data was originally captured at 1000 samples per second and subsequently median filtered and subsampled to 1 sample/minute. During the first few minutes of walking, the participants found a suitable speed and further acclimatized, and therefore, for the purposes of this study, the last 34 minutes of the captured data during walking was considered for further analysis.

\section{RESUlTS AND DiscUSSION}

A comparative analysis of the outcome from the conventional averaging approach with respect the proposed RMSD based approach is presented here.

Fig. 2 shows the original temperature data for male and female participants together with the overall average and standard deviation using the standard averaging approach typically adopted in the literature. Fig. 2 shows the data gathered from males and females together with the average temperature trend during walking for each group and the corresponding standard deviation following conventional analysis. The approach assumes a form of time-locked averaging across participants to determine temperature variations during walking following an acclimatisation period. However, it is clear that initial temperature conditions vary widely across participants, even after the acclimatisation interval, leading to an average trend with high standard deviations at each time point.

Fig. 3 shows the individual data sessions captured during summer and winter. Once again, plotting the mean for analysis only allows the researcher to analyze the influence of one factor on the captured data at a time, which for the data being considered would consist of the effect of gender on the change in foot temperature as shown in Fig. 2 and the 
effect of season/ambient conditions on the foot temperature change with time as shown in Fig. 3.

On the other hand, Fig. 4 and Fig. 5 show the results
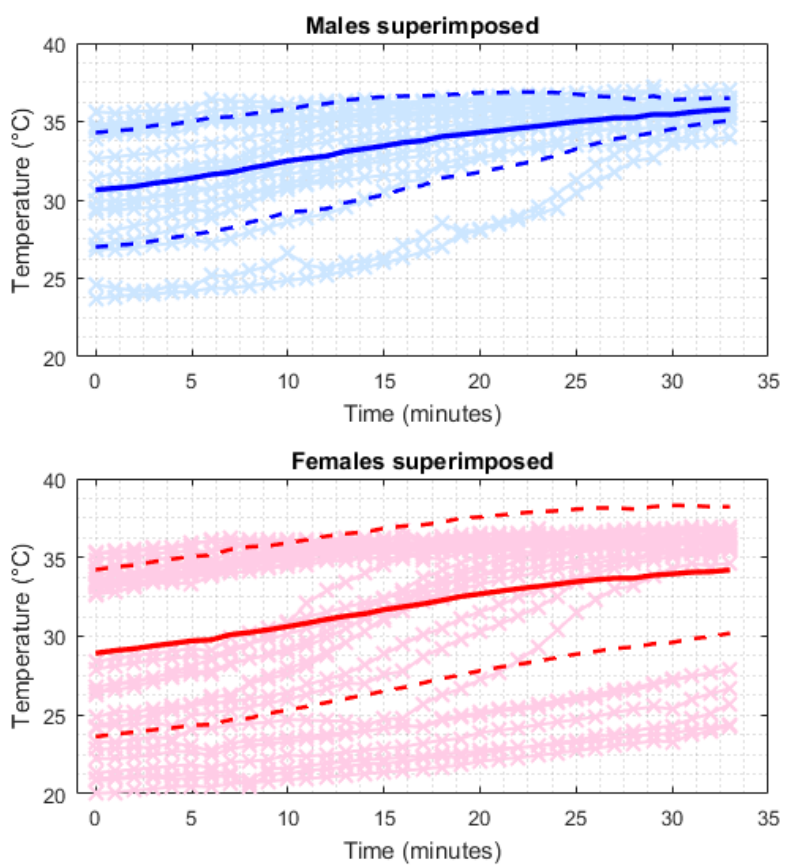

Fig. 2 (Top) Plot of individual data captured sessions for males with mean superimposed in a bold line and standard deviation superimposed in dotted lines. (Bottom) Plot of female individual data capture sessions with mean superimposed in a bold line and standard deviation superimposed in dotted lines. Data is captures from toes.
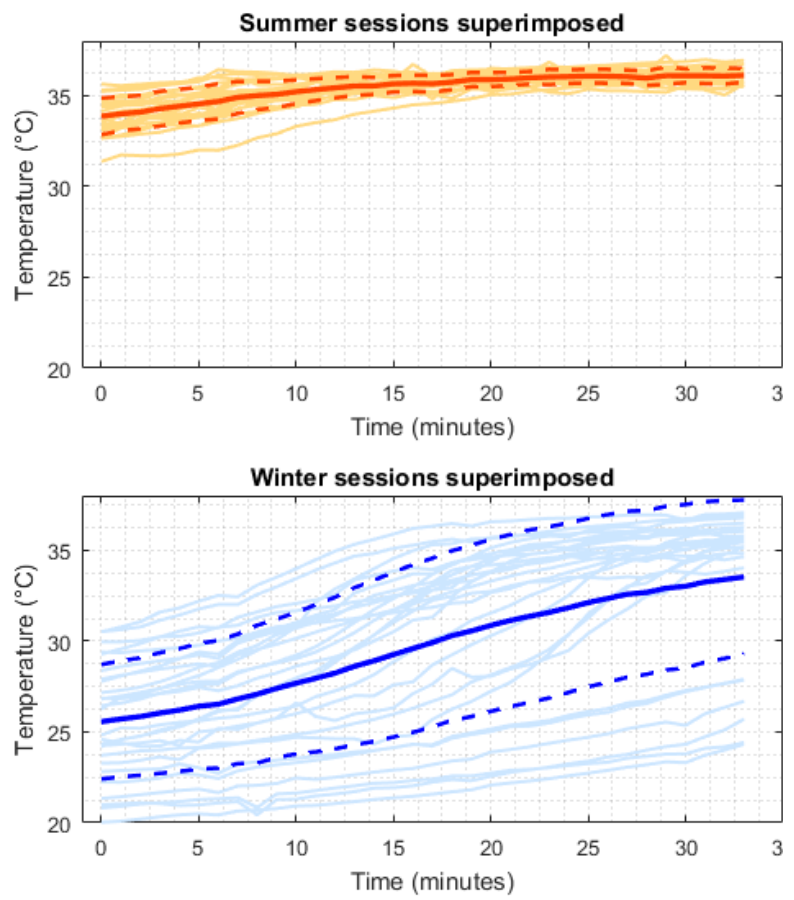

Fig. 3 (Top) Plot of individual data captured sessions during the summer season with mean superimposed in a bold line and standard deviation superimposed in dotted lines. (Bottom) Plot of individual captured data during the winter season with mean superimposed in a bold line and standard deviation superimposed in dotted lines. Data is captured from toes. obtained when the temperature data is realigned using the proposed RMSD method. Note that Fig. 4 and Fig. 5 contain the same data with different color codes to highlight how different underlying factors are captured into one continuous trend using the proposed approach. All the considered factors, in this case gender and season, can be combined into one single process. The resulting standard deviation for the proposed method in Fig. 4 and Fig. 5 is also considerably smaller than the standard deviation for the trends obtained

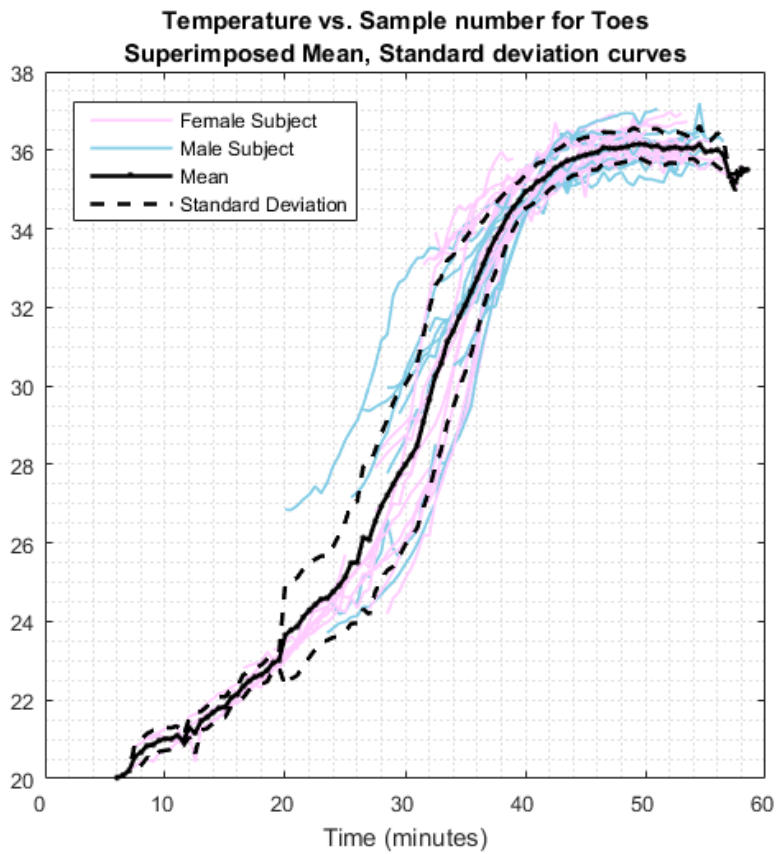

Fig. 4 All captured data sessions from toes plotted using the novel method. Data sessions are colour coded by gender of subject.

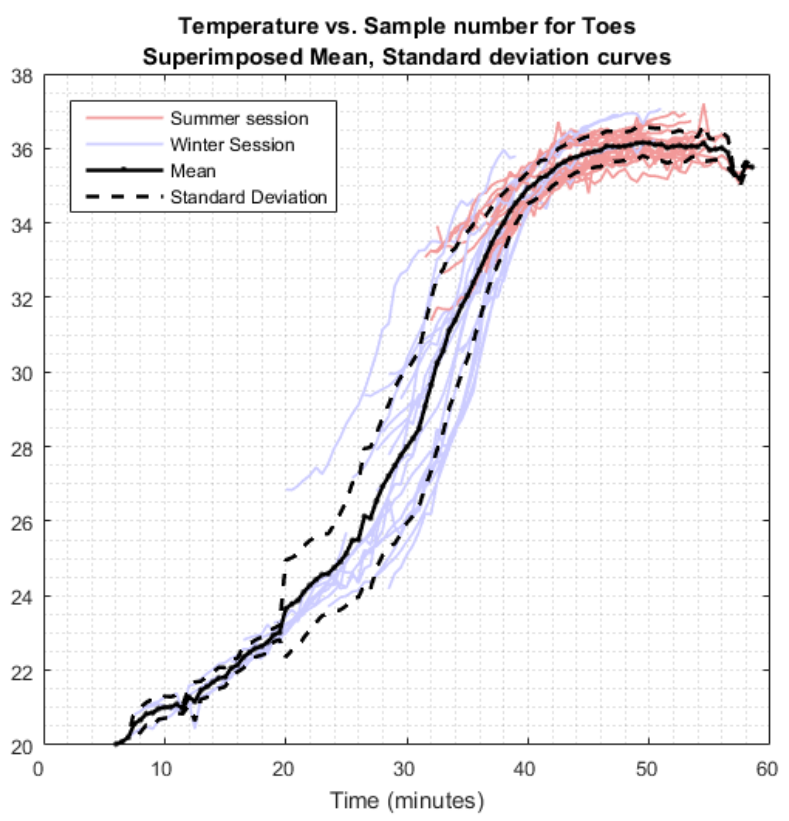

Fig. 5 All captured data sessions from toes plotted using the novel method. Data sessions are colour coded by season in which session was held. 
from the conventional approach in Fig. 2 and Fig. 3

Upon further observation of the plot of all data sessions in Fig. 4, one can notice that the initial temperature for each recording plays a significant role in the resulting position of the respective temperature curve in the determine general trend. This further indicates that discarding the starting temperature, as is done in some studies prior to the averaging step, is actually likely to further deteriorate the suitability of the extracted temperature trends. This relationship of starting temperature to trend and temperatures reached would not be visible in a plot of mean for this same study. This can also be said for number of samples at particular temperatures which is also overlooked in plotting the mean value with time. Therefore, in plotting data with time using the method proposed in this paper, one would be obtaining a more informative pattern than when plotting data using the conventional mean approach.

This overall common trend would certainly be missed in applying a conventional approach using only the mean and possibly standard deviation. This overall trend could possibly lead to an interesting physiological explanation.

This proposed approach of applying the RMSD to a set of captured data and then plotting the matched trends could be applied to any data captured from different subjects or scenarios over a period of time. Using the proposed method, all of the data captured from the different subjects or sources can be considered from one plot as well as its different trends which could be due to the different source from where the data is being captured. The different sources of the data could be due to external influences on the source which influence its response to the characteristic being monitored by the sensor from which data is being captured.

Further improvements could be implemented in the computation of the proposed method, possibly investigating the effect of increasing or decreasing the minimum threshold of the number of points used in the RMSE computations. Collecting data from more subjects and in new conditions would of course further strengthen the advantages of the proposed method.

\section{CONCLUSION}

Temperature changes for medical investigations are typically conducted by computing the mean of the number of data sessions captured over the particular time period. This approach overlooks important information such as the different individual trends of data within the specified period of time. Here we proposed and assessed a new approach for the extraction and representation of a generic temperature trends determined from multiple participants with different influencing factors. Using the proposed method, all of the data captured is plotted with time, thus all of the different aspects of the data can be characterised using one model.

While the analysis carried out in this work focused on data obtained during a walking activity, the same concept can easily be adopted to analyse temperature recordings acquired during other activities, or in relation to different body regions or medical conditions. The method may also prove useful in the analysis of other physiological signals acquired over time where to date only simple averaging procedures have been adopted to extract temporal trends from the data.

\section{REFERENCES}

[1] L.A. Houghton, B. Dawson, S.K. Maloney, "Effects of wearing compression garments on thermoregulation during simulated team sport activity in temperate environmental conditions", Journal of Science and Medicine in Sports, vol.12, no. 2, pp. 303-309, 2009.

[2] J. Rico-Sanz, W.R. Frontera, M.A. Rivera, A. Rivera-Brown, P.A Mole, C.N. Meredith, 1996. "Effects of Hyperhydration on Total Body Water, Temperature Regulation and Performance of Elite Young Soccer Players in a Warm Climate", International Journal of Sports Medicine, vol. 17, no. 2, pp. 85-91, 1996.

[3] S.R. Insler, D.I. Sessler, "Perioperative Thermoregulation and Temperature Monitoring", Anesthesiology Clinics, vol. 24, no. 4, pp. 823-837, 2006.

[4] J. Reith, S. Jorgensen, P.M. Pedersen, H. Nakamaya, L.L. Jeppesen, T.S. Olsen, H.O. Raaschou, "Body temperature in acute stroke: relation to stroke severity, infarct size, mortality, and outcome", The Lancet, vol. 347, no. 8999, pp.422-425, 1996.

[5] J.Y. Kokate, K.J. Leland, A.M. Held, G.L. Hansen, G.L., Kveen, B.A. Johnson, M.S. Wilke, E.M. Sparrow, P.A. Iaizzo, "Temperaturemodulated pressure ulcers: a porcine model", Archives of Physical Medicine and Rehabilitation, vol. 76, no. 7, pp. 666-673, 1995.

[6] P. Posada-Moreno, M.E.L. Iglesias, R.B. De Bengoa Vallejo, I. O. Soriano, I. Zaragoza-Garcia, C. Martínez-Rincón, "Influence of different bed support surface covers on skin temperature" , Contemporary Nurse, vol.39, no. 2, pp. 206-220, 2011.

[7] J.W. Scopes, I. Ahmed, "Range of Critical Temperatures in Sick and Premature Newborn Babies", Archive of Disease in Childhood, vol. 41, pp. 417-419, 1966.

[8] M. Clark, M. Romanelli, S. Reger, V. Ranganathan, J. Black, C. Dealey, "Microclimate in context. International Review. Pressure Ulcer Prevention: Pressure, Shear, Friction and Microclimate in Context. A Consensus Document”, pp. 19-25, 2010.

[9] P.N. Reddy, G. Cooper, A. Weightman, E. Hodson-Tole, N. Reeves, "An in-shoe temperature measurement system for studying diabetic foot ulceration etiology: preliminary results with healthy participants", in Procedia CIRP, vol. 49, pp. 153-156, 2016.

[10] A. Zontak, S. Sideman, O. Verbitsky, R. Beyar, "Dynamic thermography: analysis of hand temperature during exercise", Annals of Biomedical Engineering, vol. 26, no.6, pp. 988-993, 1998.

[11] R.A. Nardin, P.M. Fogerson, R. Nie, S.B. Rutkove, "Foot temperature in healthy individuals: effects of ambient temperature and age", Journal of the American Podiatric Medical Association, vol.100, no.4, pp. 258-264, 2010.

[12] J.D.J. Sandoval-Palomares, J. Yáñez-Mendiola, A. Gómez-Espinosa, J.M. López-Vela, "Portable System for Monitoring the Microclimate in the Footwear-Foot Interface", Sensors, vol. 16, no.7, pp. 1059, 2016.

[13] Y. Shimazaki, T. Matsutani, Y. Satsumoto, "Evaluation of thermal formation and air ventilation inside footwear during gait: The role of gait and fitting", Applied Ergonomics, no. 55, pp. 234-240, 2016.

[14] Y. Shimazaki, M. Murata, "Effect of gait on formation of thermal environment inside footwear", Applied Ergonomics, no. 49, pp. 55-62, 2015 . 